\title{
Polymer/organoclay nanocomposites with biodegradable aliphatic polyester and its blends: preparation and characterization
}

\author{
Sung Taek Lim ${ }^{1}$, Chung Ho Lee ${ }^{1}$, Hee Bong Kim ${ }^{1}$, Hyoung Jin Choi ${ }^{1}$ *, \\ Myung Sik Jhon ${ }^{2}$
}

${ }^{1}$ Department of Polymer Science and Engineering, Inha University, Incheon 402-751, Korea; Fax +82-32-865-5178; hjchoi@inha.ac.kr

${ }^{2}$ Department of Chemical Engineering, Carnegie Mellon University, Pittsburgh, PA 15213-3890, USA; mj3a@andrew.cmu.edu

(Received: April 15, 2004; published: May 6, 2004)

This work has been presented at the $11^{\text {th }}$ Annual POLYCHAR World Forum on Advanced Materials, January 7-10, 2003, in Denton, Texas, USA

\begin{abstract}
Synthetic biodegradable aliphatic polyester (BAP) and its blends with polyepichlorohydrin $(\mathrm{PECH})$ were processed with organophilic montmorillonite (OMMT) to make polymer/organoclay nanocomposites via a solvent casting method. Interlayer spacings of each system, measured by X-ray diffraction analysis, suggested that BAP had better affinity to clay than PECH in a competitive intercalation mechanism. The internal structures were visualized using transmission electron microscopy and were discussed in conjunction with the result of other experimental studies, such as thermogravimetric analysis and rheometry. In rheological measurements, the degree of shear-thinning increased drastically with OMMT loading, and both the storage modulus and loss modulus were found to increase with OMMT loading at all frequencies. Throughout this study, we compared the behaviours of polymer/clay nanocomposites in homo and blend systems, and examined the role of various parameters on the material characteristics of polymer/clay nanocomposites.
\end{abstract}

\section{Introduction}

The polymer/clay nanocomposite system is currently being adopted as a novel polymeric materials development due to nanoscale polymer-clay interaction, which can impart tremendous improvement over pure polymer properties. Improvements include superior physical and mechanical properties, such as gas permeability, solvent resistance, ionic conductivity, optical properties, heat resistance, decreased gas permeability, and flammability [1-7]. On the other hand, since polymer blending offers versatile routes to improve the characteristics of polymeric materials, miscible polymer blends have played important roles in the polymer industry [8]. In this paper, we hybridized these two methods to create and investigate new composite materials.

Silicate sheets mainly consisting of silicon and oxygen with a small amount of aluminium, magnesium, and other metal ions are regarded as rigid, inorganic polymers. 
Swellable clay minerals have alkali metals between the silicate sheets and can swell in polar solvents such as water. Mica-type silicates (montmorillonite, hectorite, saponite, etc.) have recently received much attention as reinforcing materials for polymers owing to their high aspect ratio and unique intercalation/exfoliation characteristics [9]. However, there exist some limitations in preparing polymer/clay nanocomposites due to the discrepancy between the hydrophilic properties of inter-layer spacing of clay and organic polymers. To improve the affinity of montmorillonite (MMT) between organic polymers and hydrophilic clay surfaces, it is necessary to modify MMT as organophilic materials by replacing the metal cations in the interlayer of silicates with various organic cation molecules.

The biodegradable aliphatic polyester (BAP) is a thermoplastic polyester synthesized from the polycondensation reaction of aliphatic glycols and aliphatic dicarboxylic acids. BAP has only ester chemical bonds and no aromatic components that may decelerate the rate of biodegradation. BAP also has physical properties similar to polyethylene and polypropylene, such as excellent processability on conventional equipment, no odour in the molten or solid state, and shows less heat of combustion than polyethylene. Since there exist some application difficulties due to its low melting temperature, low thermal stability, low molecular weight, and high material cost, improved properties can be achieved by blending BAP with conventional thermoplastics. Miscibility and rheological properties of BAP blends with linear low density polyethylene (LDPE) [10,11], poly(vinyl acetate) (PVAc) [12], and polyepichlorohydrin (PECH) [13] have been investigated, revealing that LDPE and PVAc are both immiscible, while PECH is miscible with BAP.

In this paper, we describe the preparation steps of polymer blend/clay nanocomposites via the solvent casting method using dichloromethane as a co-solvent. The type of nanocomposites (internal structure) was elucidated by X-ray diffraction (XRD). Direct visualization of the internal structure was conducted using transmission electron microscopy (TEM) and showed that the sheet-like layer structure was well preserved in the blend/clay nanocomposite systems, supporting the results of XRD. The mechanical and thermal properties of these polymer blend/clay nanocomposites were examined by tensile strength, elongation at break and thermogravimetric analysis (TGA). In addition, we examined the viscoelastic properties of polymer blend/ clay nanocomposites in several test modes. Finally, we correlated the overall rheological properties with the morphological and structural analysis of nanocomposites. From this, we systematically investigated the effect of PECH and OMMT minerals.

\section{Experimental part}

\section{Materials}

BAP $\left(M_{\mathrm{w}}=60000 \mathrm{~g} / \mathrm{mol}\right)$, a copolymer acquired from the polycondensation reaction of aliphatic glycols (ethylene glycol and 1,4-butanediol) and dicarboxylic acids (succinic acid and adipic acid), was obtained from SK Chemical (Korea). PECH $\left(M_{w}=\right.$ $700000 \mathrm{~g} / \mathrm{mol}$, Scientific Polymer Products, Inc. Ontario, NY, USA) was chosen as a counter part of BAP in our blend system, due to its miscibility with BAP and its rubbery nature, which can significantly alter the pristine characteristics of BAP. As an inorganic reinforcing material, we used organically modified montmorillonite (OMMT, Cloisite 25A), which was obtained from Southern Clay Products (Gonzales, TX, USA). The pristine $\mathrm{Na}^{+}-\mathrm{MMT}$ was treated by the manufacturer to be organophilic via a cation exchange reaction with a dimethyl, hydrogenated tallow $(\approx 65 \% \mathrm{C} 18 ; \approx 30 \%$ 
C16; $\approx 5 \%$ C14), 2-ethyhexyl, quaternary ammonium methyl sulfate. The cation exchange capacity (CEC) for Cloisite $25 \mathrm{~A}$ is $95 \mathrm{meq} / 100 \mathrm{~g}$. Hereafter, we will express the clay as OMMT to represent Cloisite 25A.

\section{Preparation of polymer blend and polymer blend/clay nanocomposites}

A series of BAP-PECH/organoclay nanocomposites was prepared via the solvent casting method using methylene chloride as a co-solvent. BAP-PECH dissolved in methylene chloride and a clay dispersion in methylene chloride were mixed together while stirring for 2 days. A vacuum oven successively removed the co-solvent. After solvent evaporation, the blend films were stored for 2 days in a vacuum oven to completely remove the residual traces of solvent. Then, the films were cut into small pieces, and compression moulded in a hot press at $140^{\circ} \mathrm{C}$ for 2 min under pressure of $1000 \mathrm{~kg} / \mathrm{m}^{2}$. To investigate the organoclay effect, we varied the clay content from 1.5 to $6.0 \mathrm{wt.} \%$ in the BAP/PECH blend system (termed as BPC1.5, BPC3.0, BPC4.5, and BPC6.0). Detailed descriptions of the composition are summarized in Tab. 1.

Tab. 1. Sample codes for the BAP-PECH/OMMT nanocomposite series

\begin{tabular}{l|lccc} 
& Sample code & $\begin{array}{c}\text { PECH } \\
\text { content } \\
\text { in wt.- } \%\end{array}$ & $\begin{array}{c}\text { Clay } \\
\text { content } \\
\text { in wt.- } \%\end{array}$ & Remarks \\
\hline BAP homopolymer & BAP & 0 & 0 & \\
BAP/clay nanocomposite & BAPC & 0 & 5 & \\
BAP-PECH blend system & BP10 & 10 & 0 & blend system \\
& BPC1.5 & & 1.5 & \\
BAP-PECH blend/clay & BPC3.0 & 10 & 3.0 & fixed PECH \\
nanocomposite & BPC4.5 & & 4.5 & content \\
& BPC6.0 & & 6.0 &
\end{tabular}

\section{Characterization}

Insertion of BAP/PECH blend into the OMMT interlayers was confirmed by XRD. Wide-angle XRD was conducted on a Philips PW-1874 X-ray crystallographic unit with $\mathrm{Cu}-\mathrm{K}_{\alpha}$ (wavelength $\lambda=0.154 \mathrm{~nm}$ ) radiation generated at $40 \mathrm{~mA}$ and $50 \mathrm{kV}$. Diffraction spectra were collected over a $2 \theta$ range of $1.5^{\circ}-10^{\circ}$ at a scan rate of $3 \%$ min. TEM photographs were obtained from a Philips CM200 UT (FEI Company, Hillsboro, Oregon, USA) operated at a voltage of $80 \mathrm{kV}$. All of the ultra-thin sections $(\approx 100 \mathrm{~nm}$ ) were microtomed using a RMC MTX ultramicrotome (UMT, Boeckeler Instruments, Inc, Tucson, AZ, USA) with a diamond knife in an epoxy moulded state, and then subjected to TEM observation without staining. The morphologies of the clay particles were investigated using a scanning electron microscope (SEM, Hitachi S-4200, Japan) with 30000 magnification at $7 \mathrm{kV}$. For the measurement, OMMT was coated with $\mathrm{Pt}$ to generate an electric current on its surface.

TGA was performed using a Perkin-Elmer TGA 7 (USA) under a nitrogen atmosphere at heating rate of $20^{\circ} \mathrm{C} / \mathrm{min}$ scanning to $900^{\circ} \mathrm{C}$. A Universal Test Machine (UTM, 
Hounsfield, H25K-S Chamber system, England) was used to measure mechanical properties including tensile modulus, strength, and elongation at break with a speed of $30 \mathrm{~mm} / \mathrm{min}$ and a $30 \times 50 \mathrm{~mm}$ sample. All measurements were conducted at $25^{\circ} \mathrm{C}$ unless otherwise specified.

The viscoelastic properties of BAP/PECH-OMMT nanocomposites were measured with a MCR 300 (Physica, Stuttgart, Germany) using a $25 \mathrm{~mm}$ diameter parallel plate geometry (PP-25) with $1 \mathrm{~mm}$ gap between two plates. Temperature was held at $160^{\circ} \mathrm{C}$. Several tests were employed to characterize the rheological properties of BAP-PECH/OMMT nanocomposite. We examined the relation between shear rate and shear stress in a steady shear mode. From this, we obtained the steady shear viscosity and the characteristic parameters in a flow curve of BAP/PECH-OMMT nanocomposites in the range of 0.001 - $10 \mathrm{rad} / \mathrm{s}$. A frequency sweep test was performed to investigate the dynamic mechanical properties of the systems in the range of $0.001-100 \mathrm{~Hz}$. The oscillatory test was performed at a fixed strain of $3 \%$, which was determined by an amplitude sweep experiment to ensure the validity of the linear viscoelasticity.

\section{Results and discussion}

In Fig. 1, we show the TEM and SEM photos of OMMT and BPC6 to assist the nanoscopic understanding of polymer/clay nanocomposites. The sheet-like particulates in the SEM photo (mid-position) consist of numerous monolayers staked in gallery form (TEM photos). OMMT is composed of very small, plate-like structures having a thickness of $1-2 \mathrm{~nm}$. Due to this colloidal size and large aspect ratio, delaminated clay possesses an extremely large surface area. It has been hypothesized that the large surface area, large aspect ratio, and good interfacial interaction are essential to produce enhanced solid-state properties. The periodic stacks of these leaflets produce a fine structure tactoid. OMMT has approximately $19.4 \AA$ interlayer gallery spacing as shown in Fig. 2. Following the insertion of polymer chains between the layers, it is observed that the $d$-spacing of the OMMT increased, similar to the result of XRD. Although direct TEM visualization does not strongly support the overall homogeneity of the polymer/clay nanocomposite due to the limited area under investigation, it still is sufficient to verify the nanoscale polymer/clay internal structure. The TEM investigation also explains the occurrence of peak broadening in XRD. Usually the interlayer spacing in intercalated polymer/clay nanocomposites is determined from the peak position, which shows an intensity maximum in the range of the

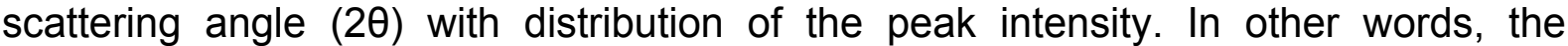
collected diffraction is distributed in a broad range of $2 \theta$. This kind of peak broadening can be directly confirmed via TEM. The TEM images show numerous types of stacked layers. This nonhomogeneity of the internal layer structure might be the origin of peak broadening in both pristine clay and polymer/clay nanocomposites. In the intercalated system, the preserved sheet structure is also found as shown in the pristine clay particle state.

The XRD spectra of OMMT and BAP/PECH-OMMT nanocomposites having different amounts of OMMT are illustrated in Fig. 2. We calculated the interlayer gallery spacing using Bragg's formula $(n \lambda=2 d \sin \theta)$. The characteristic peak of pristine OMMT occurred at $2 \theta=4.54^{\circ}$.

The peak at $4.68^{\circ}$ corresponds to the basal spacing $(1.88 \mathrm{~nm})$ of pristine OMMT. The peak value shifted to lower $2 \theta$ angles $2.84^{\circ}$ (blends system) and $1.50^{\circ}$ (PECH/OMMT nanocomposite) indicated that polymers intercalate into the OMMT layers of the BPC 
series and $\mathrm{PECH}$, respectively. These peak positions correspond to an interlayer spacing of $3.12 \mathrm{~nm}$ (for BPC series) and $5.89 \mathrm{~nm}$ (for PECH nanocomposite). In other words, polymer intercalation into OMMT layers gives rise to an increase of interlayer gallery spacing of approximately $1.24 \mathrm{~nm}$ (BPC series) and $4.01 \mathrm{~nm}$ (PECH system), when compared to pristine OMMT.
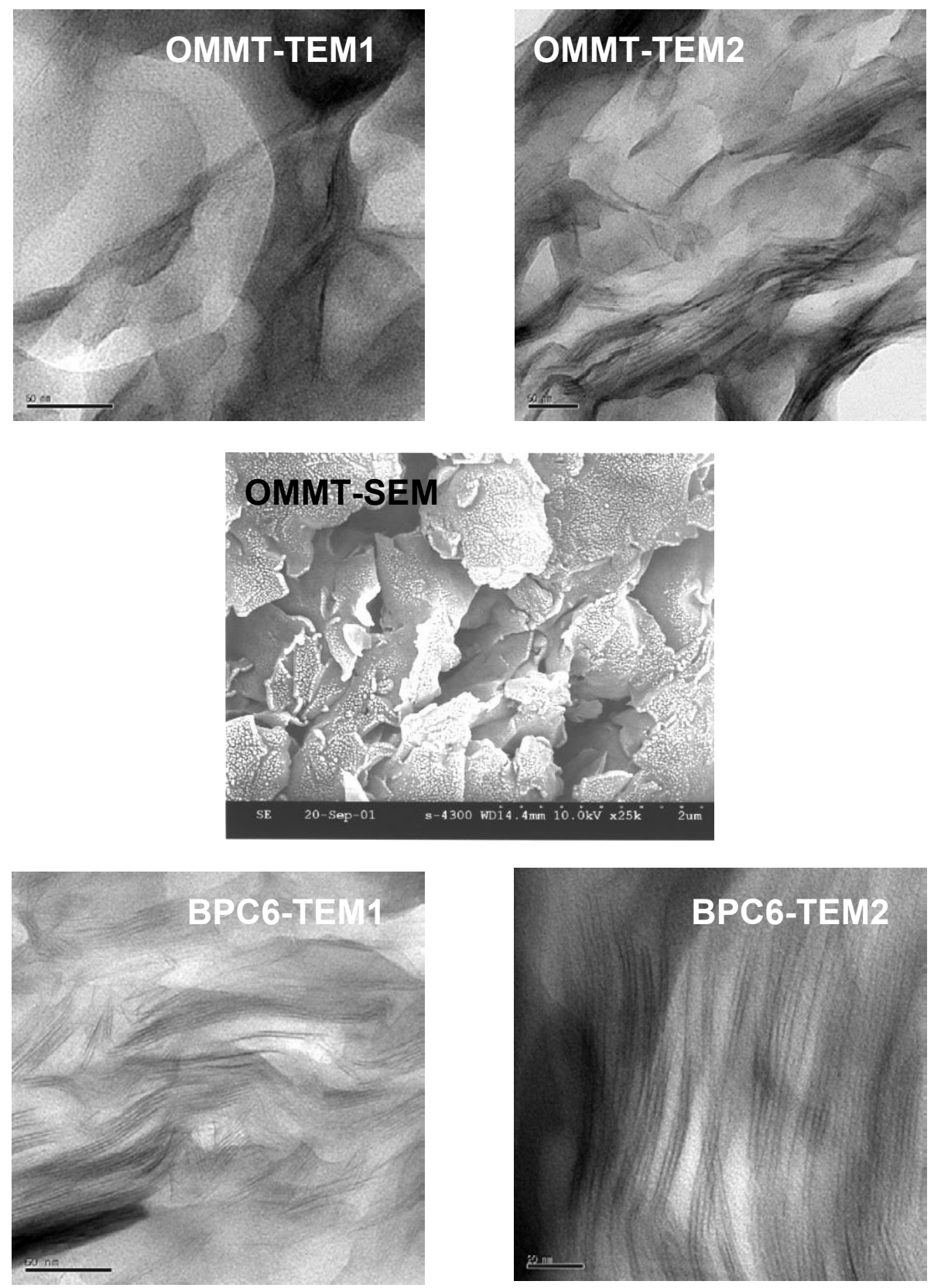

Fig. 1. TEM and SEM micrographs of pure OMMT and BAP-PECH/OMMT nanocomposites (BPC6)

Moreover, all polymer blend/clay nanocomposites have the same interlayer spacing regardless of clay loading. When we compare this result with that of simple $\mathrm{PECH} /$ OMMT nanocomposites, we find that there exists a distinct difference in intensity maximum position between polymer/clay nanocomposites and the polymer blend system. For PECH/OMMT nanocomposites, the peak position is found at much smaller $2 \theta$ position, and the interlayer spacing induced by the insertion of $\mathrm{PECH}$ is 
nearly doubled compared to the blend systems. From these, we can deduce some interesting intercalation process in polymer blend/clay nanocomposite systems. We first examined the morphology or internal change of structure for blend systems. Due to the factors affecting the affinity between polymer and clay, it is not difficult to conjecture the distinct difference between the intercalation processes for other simple polymer/clay nanocomposites. Although BAP and $\mathrm{PECH}$ are miscible in their blend systems, the structural changes accompanying the intercalating process will be different. As shown in our previous work [14], each polymer can intercalate into the interlayer spacing. However, the increase in interlayer spacing was much different (3.11 vs. $5.93 \mathrm{~nm}$ ). In the BAP-PECH/OMMT system, the overall chain insertion into the layer is dominated by BAP chains. In addition, the peak position corresponding to the blend system is almost identical to simple BAP/OMMT nanocomposite systems [14]. In our result, the structural differences, degree of affinity to clay surface, and kinematic differences in the intercalation process made only BAP clay have a better affinity to the organophilic surfaces of OMMT than PECH [15]. As expected, peak intensities increase in proportion to OMMT content. The increase in peak intensity is due to the increased amount of diffracted X-ray by OMMT. Moreover, the interlayer gallery spacing for the BPC nanocomposite series is independent of the OMMT content, which is consistent with results of other research groups [16-18].

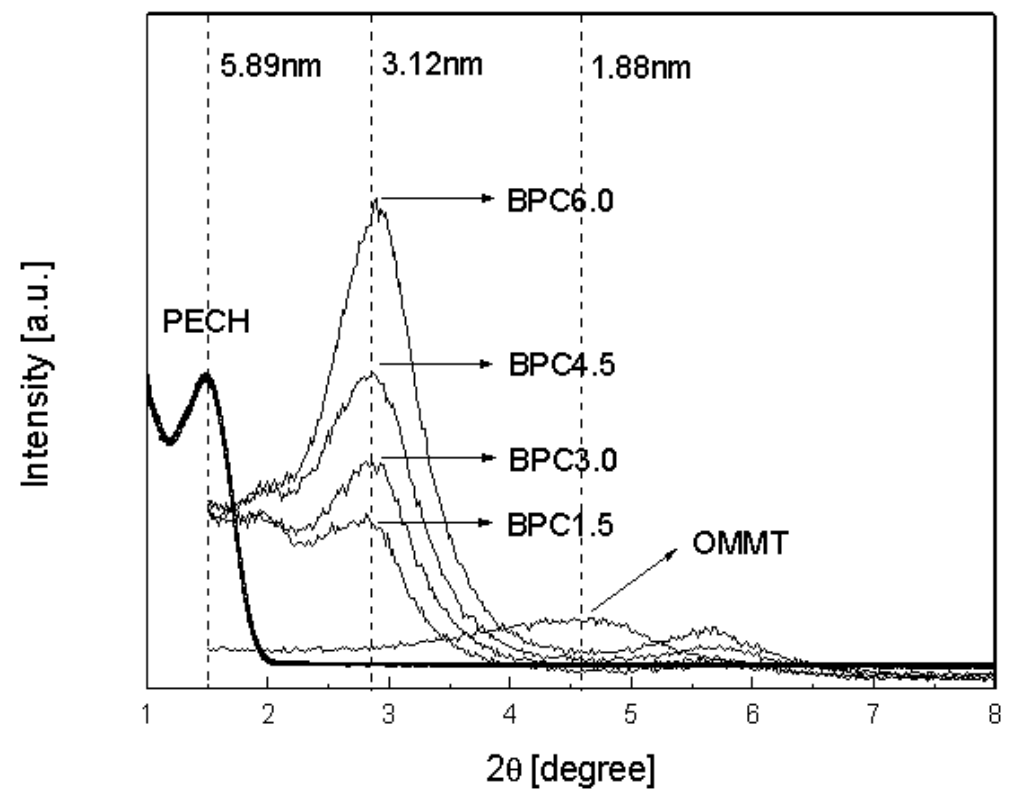

Fig. 2. XRD pattern of BAP-PECH/OMMT nanocomposite as a function of OMMT content

Several research groups have studied the rheological properties of polymer/OMMT nanocomposites to provide useful information in material processing [19-22], structural analysis, and theoretical prediction of polymer dynamics. Fig. 3 shows steady shear response and curve fitting results using the Carreau model for BAP/ PECH-OMMT nanocomposites. To examine the relationship between shear viscosity $(\eta)$ and shear rate $(\dot{Y})$, we fit the data to the Carreau model [23] given by

$\eta=\eta_{0} /\left[1+\left(t_{1} \dot{\mathrm{Y}}\right)^{2}\right]^{(1-n) / 2}$

Here, $\eta_{0}$ is the zero shear rate viscosity, which can be directly obtained from the plateau (at extremely low shear rates) as shown in Fig. 3. $t_{1}$ is a characteristic time, 
and $n$ is a dimensionless parameter, where the slope in the power-law region is given by $n-1$. Eq. (1) reduces to the Newtonian fluid constitutive relationship for $n=1$ and describes shear-thinning behaviour for $n<1$ [24].

Tab. 2. Parameters in the Carreau model (Eq. (1)) for BAP-PECH/OMMT nanocomposites

\begin{tabular}{lccc}
\hline Sample & $\eta_{0} /\left(10^{4} \mathrm{~Pa} \cdot \mathrm{s}\right)$ & $t_{1} / \mathrm{s}$ & $n$ \\
\hline BAP & 0.63 & 39.2 & 0.89 \\
BP10 & 12.8 & 55.7 & 0.59 \\
BPC1.5 & 16.9 & 78.0 & 0.58 \\
BPC3.0 & 24.7 & 72.2 & 0.55 \\
BPC4.5 & 38.1 & 83.6 & 0.51 \\
BPC6.0 & 55.4 & 66.0 & 0.47
\end{tabular}

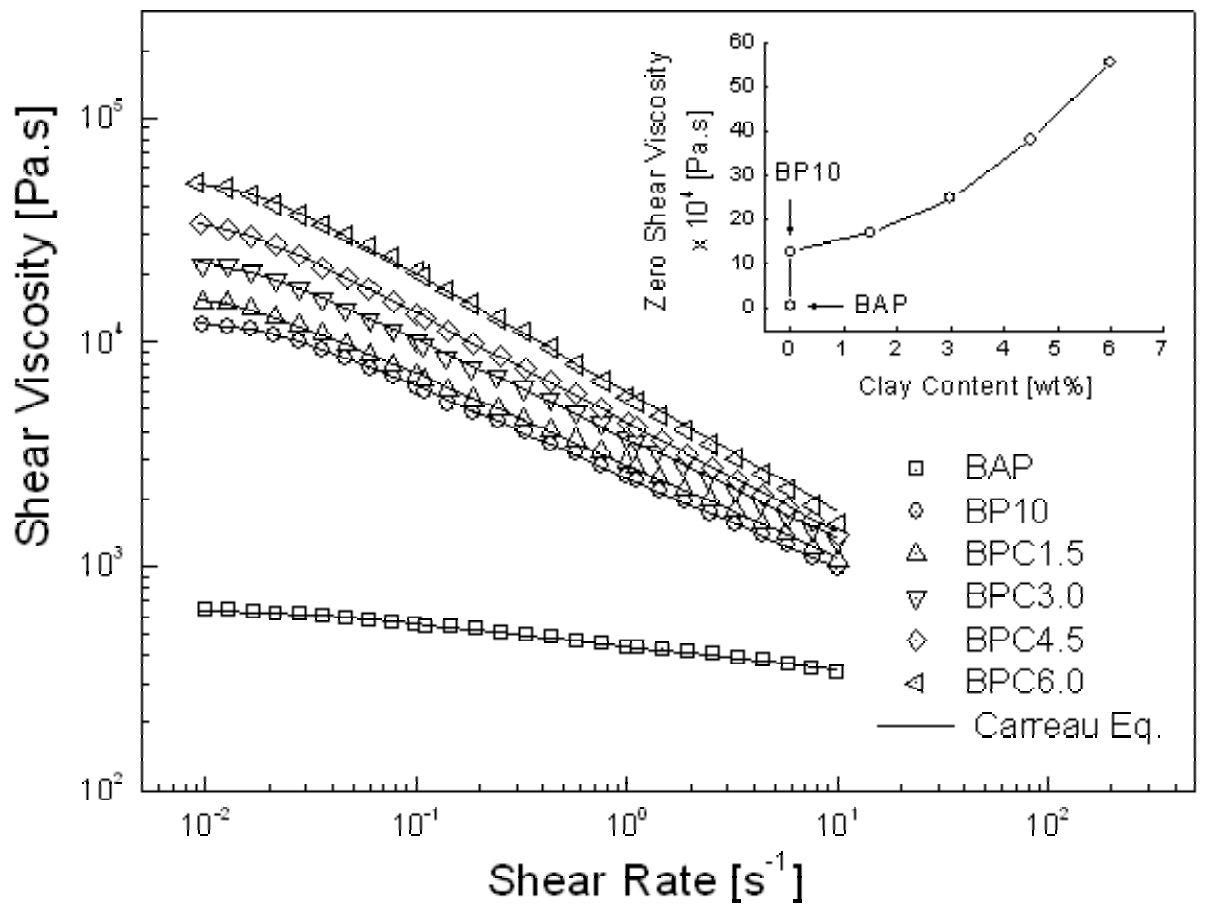

Fig. 3. Shear viscosity vs. shear rate for BAP-PECH/OMMT nanocomposites with different OMMT content. The inset illustrates the zero shear rate viscosity obtained from the Carreau model

These parameters obtained via non-linear regression are given in Tab. 2. At low shear rate, the shear viscosity is enhanced monotonically with OMMT loading. Furthermore, we find that $n$ decreases and $t_{1}$ increases with OMMT loading. The inset in Fig. 3 represents the zero shear rate viscosity (obtained from Carreau equation) as a function of clay content. From this plot, our relationship between zero shear rate viscosity and clay content is similar to previous studies $[1,25,26]$. Since BAP/PECHclay nanocomposites with higher clay loading show more pseudo-solid-like behaviour [27], effects of OMMT for this system are clearly revealed in the low shear rate region. At high shear rate, however, the viscosity and the degree of shear thinning for 
the nanocomposites are comparable with those of pristine BAP and BAP/PECH (BP10) blend.

Fig. 4(a) and (b) show dynamic oscillatory responses (storage modulus $\left(G^{\prime}\right)$, loss modulus $\left(G^{\prime \prime}\right)$ ) of BAP/PECH-OMMT nanocomposites. This oscillatory measurement is frequently used to analyze the mesoscopic structure of polymer/clay nanocomposites, and is kept within the linear viscoelastic region so as not to destroy the structure of the samples. Note that very high stresses prevent the sample from remembering its initial state after relief. These data were acquired in the range of $0.06-600 \mathrm{rad} / \mathrm{s}$ at a strain of 0.03 . At low angular frequencies (i.e., small Deborah numbers), the response is liquid-like, i.e., the loss modulus exceeds the storage modulus. In the low frequency range, known as the terminal region, in general, the double logarithmic curve of $G$ ' has a slope of two, while $G$ " has a slope of one [28].
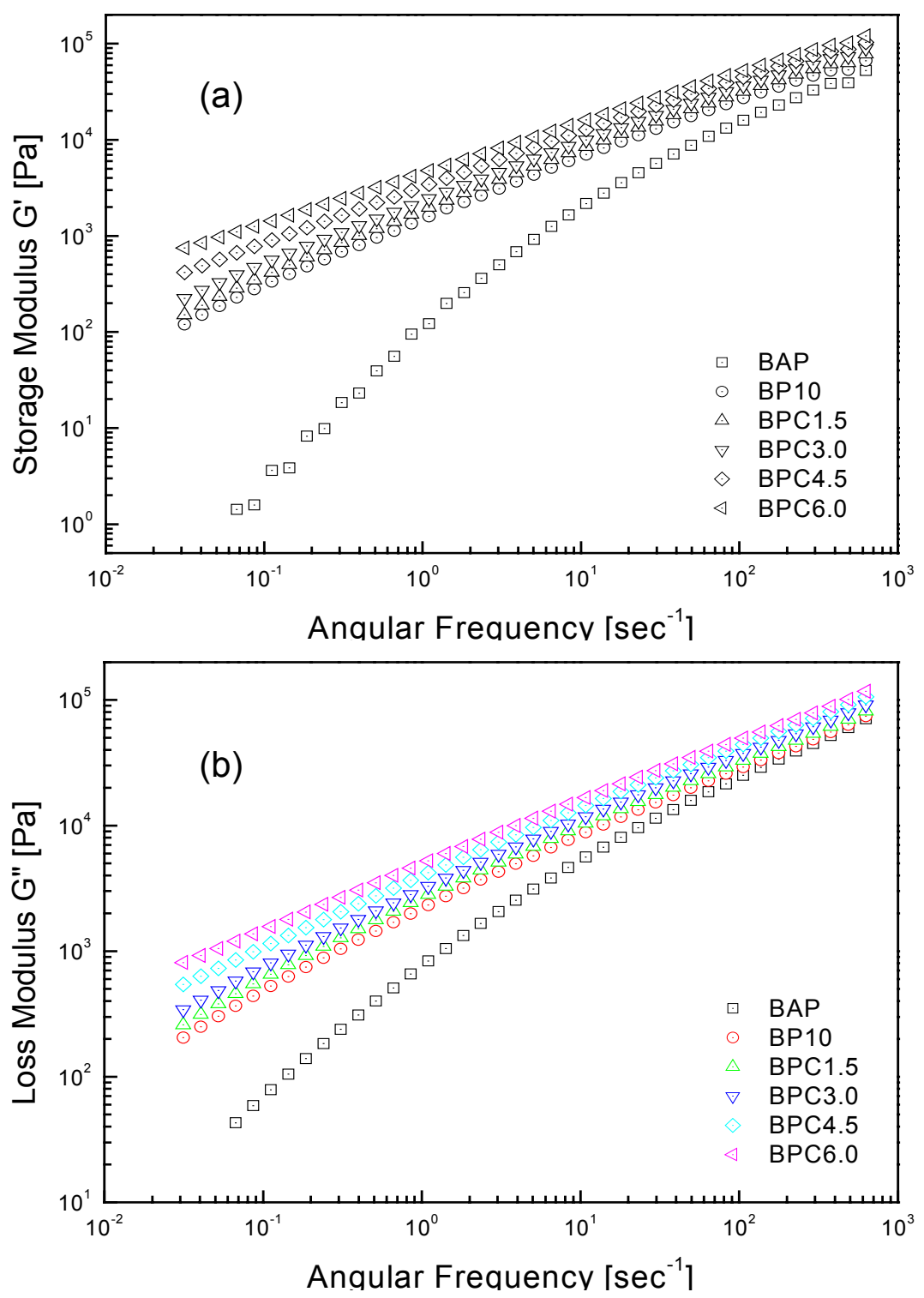

Fig. 4. Storage (a) and loss (b) moduli of BAP-PECH/OMMT nanocomposites for five different OMMT contents

The slopes of $G^{\prime}$ and $G^{\prime \prime}$ in the low shear rate region decrease with OMMT loading in Fig. 4(a) and (b), and also show a monotonic increase in the entire range of 
frequency with increasing silicate loading. In Fig. 4(a), G' shows slopes of 1.66, 0.74, $0.73,0.68,0.60$, and 0.52 , while those of $G$ " are $1.11,0.70,0.69,0.65,0.58$ and 0.53 (for BAP, BP10, BPC1.5, BPC3.0, BPC4.5 and BPC6.0), respectively, in the low frequency region. The decreasing slopes of $G$ ' and $G$ " reveal that the addition of $\mathrm{PECH}(10 \mathrm{wt.}$ \%) leads the nanocomposites to non-terminal behaviour and increasing OMMT loading in BAP/PECH blends gives rise to more severe nonterminal behaviour in the low frequency region. Moreover, $G$ ' became less dependent on frequency with OMMT loading in the low frequency region. This means that more solid-like characteristics are induced by nanoscopic dispersion of organoclay compared to the non-clay systems (BAP and BP10) $[16,19]$. The resultant frequency dependences of $\tan \delta\left(\equiv G^{\prime \prime} / G^{\prime}\right.$, not shown here) decrease with frequency for the whole series of nanocomposites. At low frequencies, the behaviour of BAP-PECH/ OMMT nanocomposites is more liquid-like having higher tan $\delta$ than in the high frequency region [29]. The absolute values ranging from 2 to 1 imply that the dispersed particles are weakly associated with each other except for pure BAP (nonassociated case) [30]. tan $\delta$ is also dependent on the clay content. The crossover point $(\tan \delta=1)$ frequencies are 23, 82, 177 and $228 \mathrm{rad} / \mathrm{s}$ for BPC1.5, BPC3.0, BPC4.5, and BPC6.0. For BP10 and BAP, the crossover point is not appearing in the tested region. Comparing with BPC1.5, 3.0, 4.5, and 6.0, it is reasonable to conjecture that the crossover points of BP10 and pure BAP exist at higher frequencies. In summary, we can conclude that BAP/PECH-OMMT nanocomposites behave more solid-like as OMMT content increases.

The nanoscopic incorporation of inorganic particles can affect the thermal properties of the polymer matrix. This change in thermal properties can explain the role of filling materials at elevated temperatures. Fig. 5 shows TGA results for BAP-PECH/OMMT nanocomposites having various OMMT contents.

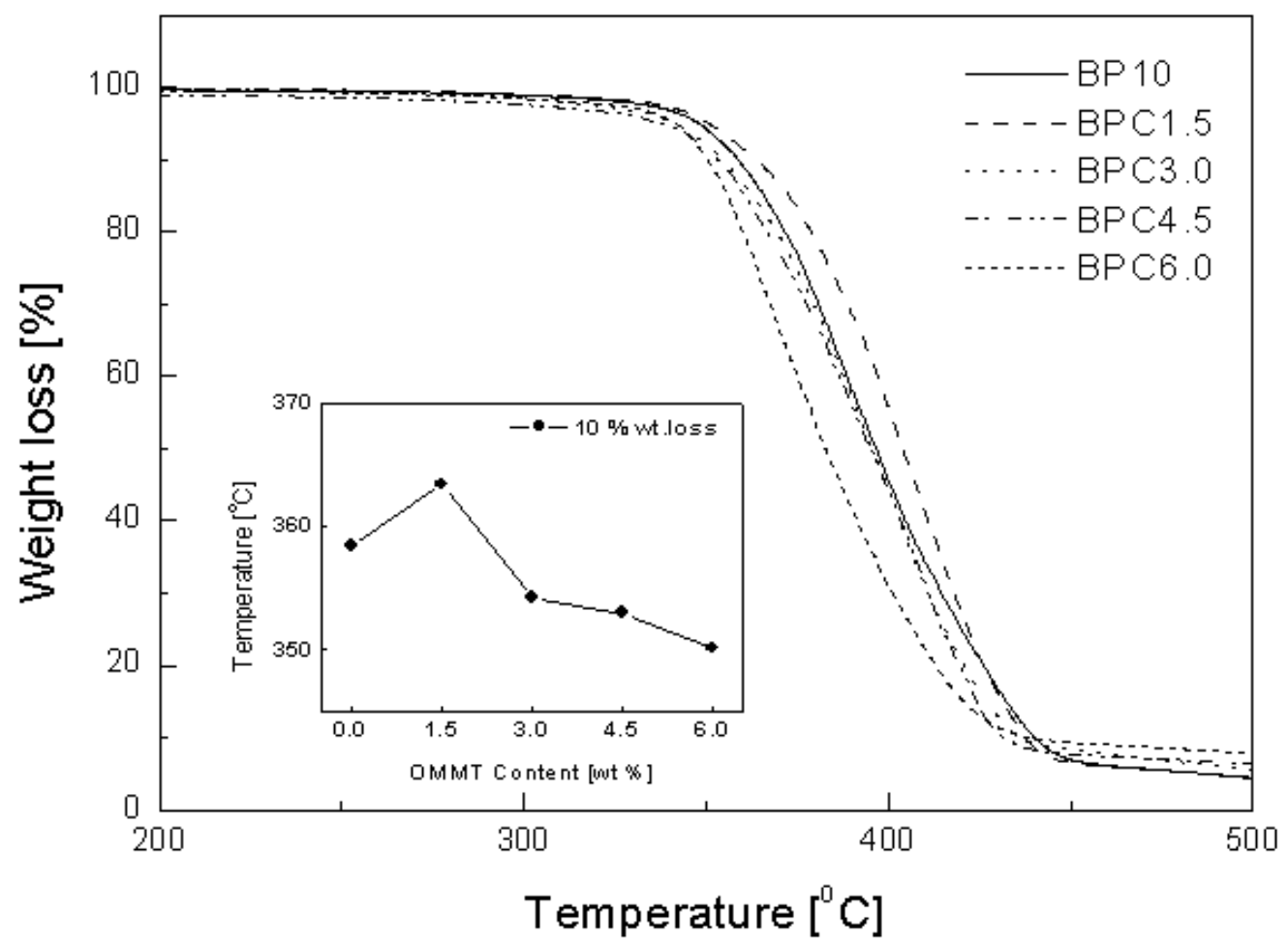

Fig. 5. TGA diagram of BAP-PECH/OMMT nanocomposites with five different OMMT contents. The inset shows the $10 \%$ weight loss temperature $v$ s. OMMT content 
The onset point of thermal degradation corresponding to $10 \%$ weight loss increases up to $1.5 \mathrm{wt} . \mathrm{\%}$ of OMMT loading and then decreases with increasing OMMT loading. These onset points are plotted in the inset of Fig. 5. This kind of characteristic thermal behaviour with a maximum was also reported previously [31-33]. The aggregation or poor dispersion of the additional OMMT [31,33] causes the decrease in thermal properties. This abnormal trend in the blend/OMMT nanocomposites might also be caused by the different, selective intercalation mechanism. Namely, the increase of clay content could enhance the nanolevel incorporation with only BAP chains, except for $\mathrm{PECH}$. This selective incorporation becomes more distinct with the addition of clay. As shown in Fig. 5, the deterioration of thermal properties increased with clay loading over a specific content, which could give rise to the enhancement in thermal properties. As the clay loading increases, the excluded PECH portion could facilitate thermal conduction. But these kinds of deductions can be separated with the enhancement of viscoelastic properties via clay loading, because the measured conditions are fundamentally different. In rheological properties, the lower temperature regions cannot be affected by the selective intercalated internal structure. It means that the dispersion quality of OMMT particles in the melt state can be considered as homogeneous. But under extremely severe conditions, such as high temperature, which could give rise to thermal decomposition, the characteristic role of insulating nanolevel materials can give rise to the reverse trend in the blend/clay nanocomposite system due to the selective intercalation mechanism. This result can also be related with that of a mechanical test, which will be explained later. Despite the reverse effect in thermal decomposition temperature, the residual weight beyond $500^{\circ} \mathrm{C}$ increases with OMMT loading because the OMMT does not burn at high temperature, as shown by the increase of residual char formation.

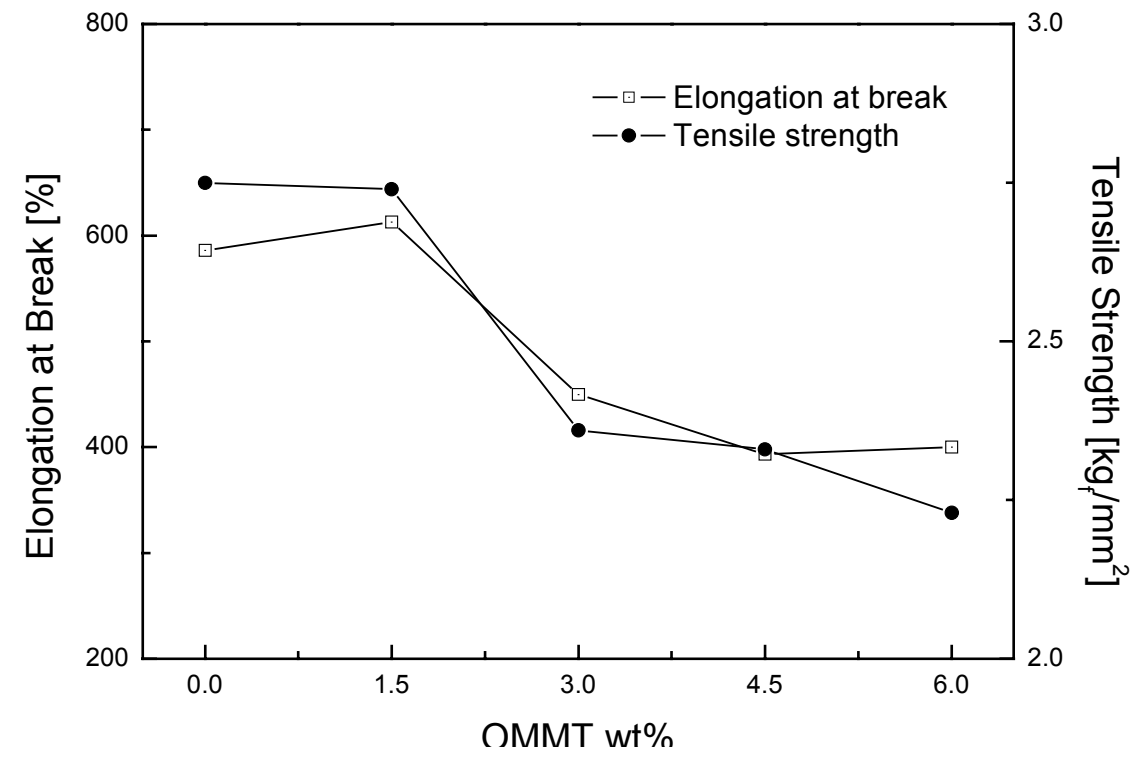

Fig. 6. Tensile strength and elongation at break vs. temperature for BAP-PECH/ OMMT nanocomposites

Mechanical properties were measured to evaluate the reinforcement effect for the OMMT. Fig. 6 shows the tensile strength and elongation at break. The tensile modulus for the BPC system is dramatically improved as the amount of OMMT increases $[34,35]$. This phenomenon is related to the increasing modulus in oscillatory tests and the relaxation modulus in stress relaxation tests. On the other hand, 
the tensile strength and elongation at break values for BPC systems increase up to 1.5 wt.- $\%$ of OMMT content, but decrease afterwards. With 3.0 wt.- $\%$ OMMT content, the tensile strength and elongation at break decrease to $2.36 \mathrm{~kg} / \mathrm{mm}$ and $450 \%$, respectively. The collapse of the mechanical properties is attributed to the aggregation of the OMMT nanolayers [31,33]. Similar results on polyethylene/ $\mathrm{Na}^{+}-\mathrm{MMT}$ nanocomposites are reported by Liao et al. [36]. Based on the result, we can systematically describe the role of clay in nanocomposites. Due to the nanoscopic incorporation of polymer chains, the solid-like transition of rheological properties can be obtained in the melt state, despite the agglomerate formation, which does not affect the overall flow characteristics. But internal imperfections become significant under extreme conditions (i.e., thermal decomposition conditions) or mechanical service environment (i.e., elongation), with the help of selective intercalated internal structures [37].

\section{Conclusion}

We investigated the internal structure, linear viscoelastic behaviour, thermal and mechanical behaviour of BAP/PECH-OMMT nanocomposites prepared via the solvent casting method. Investigation by XRD revealed that only BAP chains intercalate into the OMMT inter-space layers, suggesting that BAP has higher affinity to the OMMT inter-space layer surface than $\mathrm{PECH}$. Rheological measurements confirmed that BAP/PECH-OMMT nanocomposites exhibit more solid-like behaviour with increasing OMMT loading. The shear viscosity also rapidly decreases with OMMT loading due to the OMMT tactoid alignment in the direction of shear flow. The zero shear rate viscosity, however, is also increased with OMMT loading. For various OMMT systems, thermal decomposition temperature, tensile strength, and elongation at break have either a maximum or critical values at 1.5 or $3.0 \mathrm{wt} .-\%$ OMMT loading. Therefore, it is reasonable to conclude that the aggregation or poor dispersion of the additional OMMT loading acts as limiting factor in the enhancement of material properties. Namely, the linear viscoelastic properties are related to the mesoscopic structure of the nanocomposite. To clarify the precise internal mechanisms, further research on polymer-clay nanocomposites is desirable.

Acknowledgement: This study was supported by Korea Research Foundation Grant (KRF-2002-041-D00193).

[1] Galgali, G.; Ramesh, C.; Lele, A.; Macromolecules 2001, 34, 852.

[2] Choi, H. J.; Kim, S. G.; Hyun, Y. H.; Jhon, M. S.; Macromol. Rapid Commun. 2001, 22, 320.

[3] Kojima, Y.; Usuki, A.; Okada, A.; Kurauchi, T.; Kamigato, O.; J. Mater. Res. 1993, 8, 1174.

[4] Gilman, J. W.; Appl. Clay Sci. 1999, 15, 31.

[5] Kim, J. W.; Liu, F.; Choi, H. J.; J. Ind. Eng. Chem. 2002, 8, 399.

[6] Sur, G. S.; Lyu, S. G.; Chang, J. H.; J. Ind. Eng. Chem. 2003, 9, 58.

[7] Messersmith, P. B.; Giannelis, E. P.; J. Polym. Sci., Polym. Chem. 1995, 33, 1047. 
[8] Pathak, J. A.; Colby, R. H.; Kamath, S. Y.; Kumar, S. K.; Stadler, R.; Macromolecules 1998, 31, 8988.

[9] Okada, K.; Mitsunaga, T.; Nagase, Y.; Korea-Australia Rheol. J. 2003, 15, 43.

[10] Kim, J.; Kim, J. H.; Shin, T. K.; Choi, H. J.; Jhon, M. S.; Eur. Polym. J. 2001, 37, 2131.

[11] Sung, J. H.; Hyun, Y. H.; Kwon, D. H.; Choi, H. J.; J. Ind. Eng. Chem. 2002, 8, 28.

[12] Shin, T. K.; Kim, J. H.; Choi, H. J.; Jhon, M. S.; Polymer 1999, 40, 6873.

[13] Kim, J.; Shin, T. K.; Choi, H. J.; Jhon, M. S.; Polymer 1999, 40, 4135.

[14] Lim, S. T.; Hyun, Y. H.; Choi, H. J.; Jhon, M. S.; Chem. Mater. 2002, 14, 1839.

[15] Lim, S. K.; Kim, J. W.; Chin, I.; Kwon, Y. K.; Choi, H. J.; Chem. Mater. 2002, 14, 1989.

[16] Ren, J.; Silva, A. S.; Krishnamoorti, R.; Macromolecules 2000, 33, 3739.

[17] Vaia, R. A.; Jandt, K. D.; Kramer, E. J.; Giannelis, E. P.; Macromolecules 1995, 28, 8080.

[18] Vaia, R. A.; Giannelis, E. P.; Macromolecules 1997, 30, 7990.

[19] Krishnamoorti, R.; Giannelis, E. P.; Macromolecules 1997, 30, 4097.

[20] Hyun, Y. H.; Lim, S. T.; Choi, H. J.; Jhon, M. S.; Macromolecules 2001, 34, 8084.

[21] Lim, Y. T.; Park, O. O.; Rheol. Acta 2001, 40, 220.

[22] Hoffmann, B.; Kressler, J.; Stöppelmann, G.; Friendrich, C.; Kim, G. M.; Colloid Polym. Sci. 2000, 278, 629.

[23] Carreau, P. J.; de Kee, D. C. R.; Chhabra, R. P.; 'Rheology of Polymeric Systems', Hanser Publishers, Munich 1997.

[24] Lim, S. T.; Choi, H. J.; Jhon, M. S.; J. Ind. Eng. Chem. 2003, 9, 51.

[25] Krishnamoorti, R.; Vaia, R. A.; Giannelis, E. P.; Chem. Mater. 1996, 8, 1728.

[26] Giannelis, E. P.; Appl. Organomet. Chem. 1998, 12, 675.

[27] Krishnamoorti, R.; Beall, G. W.; 'Polymer-clay nanocomposites', John Wiley \& Sons, New York 2000.

[28] Gupta, R. K.; 'Polymer and composite rheology', Marcel Dekker, New York 2000.

[29] Baird, D. G.; Collias, D. I.; 'Polymer processing principles and design', John Wiley \& Sons, New York 1998.

[30] Rohn, C. L.; 'Analytical polymer rheology; structure processing properties relationships', Hanser Publisher, Munich 1995.

[31] Agag, T.; Koga, T.; Takeichi, T.; Polymer 2001, 42, 399.

[32] Delozier, D. M.; Orwoll, R. A.; Cahoon, J. F.; Johnston, N. J.; Smith, J. G.; Connell, J. W.; Polymer 2002, 43, 813.

[33] Yang, Y.; Zhu, Z.; Yin, J.; Wang, X.; Qi, Z.; Polymer 1999, 40, 4407.

[34] Cho, J. W.; Paul, D. R.; Polymer 2001, 42, 1083.

[35] Liu, L.; Qi, Z.; Zhu, X.; J. Appl. Polym. Sci. 1999, 71, 1133.

[36] Liao, B.; Song, M.; Liang, H.; Pang, Y.; Polymer 2001, 42, 10007.

[37] Lim, S. T.; Hyun, Y. H.; Lee, C. H.; Choi, H. J.; J. Mater. Sci. Lett. 2003, 22, 299. 